\title{
EVALUACIÓN DE LA ASOCIACIÓN DE UNA BUENA HIGIENE ORAL CON HÁBITOS DE AUTOCUIDADO ADECUADOS EN ESCOLARES VINCULADOS A DOS CONCENTRACIONES ESCO- LARES DE BUCARAMANGA Y SU ÁREA METROPOLITANA
}

\author{
Sonia Constanza Concha Sánchez \\ Odontóloga, U. Santo Tomás, Especialista en Educación y Comunicación para la Salud y el Bienestar, U. Industrial de Santander, \\ Docente, U. Santo Tomás y U. Industrial de Santander
}

Autor responsable de correspondencia: Sonia Constanza Concha S.

Correo electrónico: sococosa@yahoo.com

\begin{abstract}
RESUMEN
Objetivo: Identificar la asociación de una buena higiene oral con hábitos de autocuidado en escolares vinculados a dos concentraciones escolares de Bucaramanga y su área metropolitana.

Materiales y métodos: Mediante un estudio observacional analítico de corte transversal, se evaluaron 291 escolares de dos instituciones educativas. Las variables se describieron en forma univariada y bivariada, para analizar la posible asociación del nivel de higiene oral con variables sociodemográficos y de hábitos de autocuidado mediante el test de Chi cuadrado y el test exacto de Fisher y para variables cuantitativas, la prueba t de student. Para el análisis multivariado se aplicó una regresión binomial considerando como variable de interés buena higiene oral y las variables sociodemográficas y de autocuidado como posibles variables explicatorias.

Resultados: Se encontró una relación 14 veces mayor (IC 95\% 3.47-59.9) de tener buena higiene oral entre los escolares que adoptan más de tres hábitos de autocuidado que entre aquellos que adoptan tres o menos con diferencias estadísticamente significativas $\mathrm{p}<0.0001$ y relación $46 \%$ mayor de tener mala higiene oral en escolares matriculados en primero y segundo grado de primaria y $9 \%$ mayor en escolares de tercero cuarto y quinto de primaria, comparados con los de preescolar . Conclusión: El abordaje de programas de promoción de la salud, que consideran la relación del número hábitos de autocuidados adecuados adoptados por los escolares con el hábito de higiene oral, pueden constituirse una estrategia que permite integrar acciones que fomentan la construcción de una cultura de la salud, promoviendo efectivamente la salud oral. [Concha SC. Evaluación de la asociación de una buena higiene oral con hábitos de autocuidado adecuados en escolares vinculados a dos concentraciones escolares de Bucaramanga y su área metropolitana. Ustasalud Odontología 2005; 4: 91 - 98]
\end{abstract}

Palabras clave: Promoción de la salud, Educación en salud, Escolares, Hábitos de autocuidado.

\section{EVALUATION OF THE ASSOCIATION OF A GOOD ORAL HYGIENE WITH HABITS OF SELF-CARE IN STUDENTS FROM TWO SCHOOLS OF BUCARAMANGA AND ITS METROPOLITAN AREA}

\begin{abstract}
Objective: To identify the associations of a good oral hygiene with adequate self-care habits in school children of two Bucaramanga scholar institutions.

Material and methods: An observational analytical cross-sectional study was done, in two-hundred-ninety-one school children. The socio demographic variables and self-care habits were evaluated. Chi square and t students test were run. A binomial regression was done for the multivariate analysis.

Result: A relationship fourteen times higher was established in school children with at least four self-care habits compared to those children that have three or less habits.

Conclusion: Health promotion programs that considered the association between the number of self-care habits can be used as a strategy that will allow to build a complete picture that will include people, health culture and adequate oral health promotion.
\end{abstract}

Key words: Health promotion, Health education, School children, Self-care habits.

Recibido para publicación: 30 de junio de 2005. Aceptado para publicación: 10 de octubre de 2005. 


\section{INTRODUCCIÓN}

Mejorar las habilidades personales es una de las estrategias propuestas en la carta de Ottawa para hacer promoción de la salud. ${ }^{1}$ La adopción de hábitos y estilos de vida saludables son acciones y decisiones que asumen las personas y que están determinados, en la mayoría de los casos, por las habilidades de los individuos, por ello es fundamental investigar en estos aspectos. Sin embargo, cimentar la promoción de la salud en abordajes fundamentados en los estilos de vida tiene opositores. ${ }^{1}$

Es indudable, que la poca investigación en prevención permanece como el principal impedimento para determinar acciones en salud pública efectivas y es quizás el reflejo de la incapacidad de traducir el conocimiento en acciones que fomenten la salud. ${ }^{2}$

Las instituciones educativas son consideradas como los lugares más apropiados para promocionar la salud de los niños y las niñas, ya que la escuela puede proveer un ambiente para mejorar la salud, la autoestima, los comportamientos y las habilidades para vivir. ${ }^{3}$

Por muchos años, los programas de base escolar promocionaban la salud a través de actividades de educación en salud de tipo tradicional que han tenido efectos mínimos y a corto plazo; revisiones exhaustivas sobre los programas de educación, particularmente de salud oral, mostraban que los programas de base escolar fueron relativamente ineficientes. ${ }^{3-5}$

Al parecer, el solo conocimiento sobre salud no es suficiente para que los escolares adopten buenas prácticas de autocuidado oral. ${ }^{4}$ Revisiones sistemáticas adicionales, sugieren que la promoción de la salud en las escuelas, que se llevan a cabo de forma comprensiva podrían beneficiar la salud oral de los escolares. ${ }^{3}$

Abordar los programas de educación en salud oral en forma aislada no se constituye en el mejor abordaje desde el punto de vista promocional y preventivo; el manejo simultaneo de varias condiciones de salud relacionadas, podría ser más efectivo y eficiente, evitando en el mejor de los casos la duplicación de esfuerzos y en el peor, mensajes contradictorios para el público receptor de este tipo de programas. ${ }^{1}$
El desarrollo de programas orientados a fomentar la salud debe fundamentarse en una visión integral de las personas, considerando, los aspectos físicos, mentales, sociales, culturales y ambientales que integran y rodean el individuo. ${ }^{5}$

Una nueva visión de salud en este nuevo siglo, hace evidente la necesidad de enseñar más acerca del efecto de los factores comportamentales, para que las personas puedan empezar a hacer cambios, ya que hay una gran brecha entre las prácticas y las ganancias potenciales que se obtienen cuando se asumen comportamientos saludables. ${ }^{6}$

El estilo de vida representa patrones sociales y del comportamiento. ${ }^{7}$ La adopción de estilos de vida saludables y de hábitos de autocuidados deberían inculcarse en periodos tempranos de la vida, con el propósito de aumentar la probabilidad de que estos se mantengan, por tal razón iniciar programas de educación en salud orientados a fortalecer comportamientos saludables deberían iniciarse en el hogar y/o en las instituciones de educación primaria.

Para lograr un buen nivel de salud oral es necesario que las personas desarrollen patrones de comportamiento adecuados de tal forma que éstos se conviertan en componentes integrales de su cuidado dental diario, motivando a la higiene oral. ${ }^{8}$

Recientemente, se ha observado alguna evidencia de la etiología del comportamiento común en odontología y se ha encontrado que los comportamientos en salud general están asociados con los comportamientos en salud dental, al menos en algún grado. ${ }^{9}$

La negligencia en hábitos de cuidado en salud dental, se ha relacionado con un amplio complejo de problemas en los estilos de vida de los adolescentes; ;0,11 también, se ha observado que los comportamientos relacionados con salud oral han mostrado estar asociados con fumar, consumo de alcohol y actividad fisica en la población adulta. ${ }^{9}$ Así mismo, se ha observado que las enfermedades crónicas como las alteraciones cardiovasculares, el cáncer e incluso la caries dental están estrechamente ligadas a condiciones relacionadas a estilo de vida, factores sicológicos y condiciones sociales. ${ }^{1,10}$ Dentro de la litera- 
tura revisada, no se encontraron artículos que relacionaran hábitos o estilos de vida con los hábitos de cuidado dental en la población escolar.

Con lo expuesto se podría pensar que la educación en hábitos de autocuidado orientados a promover la higiene oral en los escolares, no debe abordarse como un actividad aislada, sino como un programa que integra el conjunto de hábitos de cuidado personal, fortaleciendo la alternativa de crear una cultura en salud. ${ }^{1}$ El diagnóstico de los hábitos de autocuidado en los escolares se podría constituir en la estrategia inicial que orientaría los programas que promueven la salud general y oral.

Considerando lo anterior, el objetivo de la presente investigación fue identificar la asociación de una buena higiene oral con hábitos de autocuidado adecuados en escolares vinculados a dos concentraciones escolares de Bucaramanga y su área metropolitana.

\section{MATERIALES Y MÉTODOS}

Se realizó un estudio observacional analítico de corte transversal. ${ }^{12-14} \mathrm{El}$ universo, estuvo integrado por 200 escolares de transición a quinto de primaria vinculados a una concentración educativa rural del municipio de Piedecuesta perteneciente al estrato socioeconómico 1 (constituida fundamentalmente por población desplazada 0 destechada) y 240 niños y niñas pertenecientes a los grados transición a tercero primaria de una institución educativa urbana de los estratos socioeconómicos 2 y 3 del municipio de Bucaramanga y que fueron evaluados durante el segundo semestre del 2004.

El tamaño de muestra se calculó mediante la rutina sample-size para estudios de corte transversal, del programa EPI-INFO v.6.04 ${ }^{15}$ con base en las siguientes especificaciones de diseño: Prevalencia de mala higiene oral en el grupo no expuesto $10 \%$ y en expuestos de $30 \%{ }^{16}$, OR (Odds Ratio) 3.86, R.R. (Riesgo Relativo) 3.0, confianza de 99.9\% y poder del $80 \%$; lo que permitió estimar que una muestra aproximada de 290 escolares detectaría asociaciones estadísticamente significativas.

Se realizó un muestreo por conveniencia; considerando como criterios de inclusión escolares vinculados a las instituciones ya mencionadas y que aceptaron participar en el estudio; se consideró como criterio de exclusión que el escolar no estuviese presente al momento del examen.

Se recolectaron variables sociodemográficas como edad, género, grado escolar, institución a la que estaban vinculados y como hábitos de cuidado personal se analizaron además del número de conductas de autocuidado asumidos por el escolar, aquellos como higiene de la indumentaria, del cabello, de uñas y manos, presencia de problemas de la piel e higiene oral, de acuerdo a los criterios establecidos para el índice cuantificado de placa (ICP) ${ }^{17}$ que se registraron como variables categóricas dicotómicas (inadecuado-adecuado).

Cada institución fue notificada con anterioridad y a cada una de ellas, se les entregó el plan de trabajo con lo cual se obtuvo la aprobación del director. Previa recolección de la información se realizó un entrenamiento a los encuestadores (estudiantes de la asignatura de promoción y prevención I) en los procedimientos y manejo de formatos de recolección de datos; se hizo prueba piloto con ocho escolares voluntarios, con el fin de verificar el diligenciamiento de la encuesta por parte de los examinadores y estandarización de criterios.

Finalmente, se procedió a recolectar las variables de la siguiente manera: Los evaluadores visitaron la institución educativa el día establecido e hicieron la evaluación de cada escolar en el salón de clase; registraron inicialmente las conductas de autocuidado general, posteriormente proporcionaban la solución reveladora y examinaban cavidad oral, seleccionado tres estructuras dentarias del maxilar superior que evaluaron por vestibular $\mathrm{y}$ tres inferiores por lingual de acuerdo a los criterios establecidos en el ICP ${ }^{17}$ y registraron sus resultados en el formato diseñado para tal fin.

La base de datos se elaboró en Excel ${ }^{18}$ por duplicado se exportaron al paquete estadístico EPI-INFO v.6.04 15 y mediante la rutina validate se corroboró la calidad de la digitación; finalmente el análisis estadístico se efectuó en el paquete estadístico STATA 8.0. ${ }^{19}$

El análisis estadístico se realizó de la siguiente manera: En el análisis univariado, se describió la población de estudio en todas sus variables aplicando medidas de resumen que se organizaron en tablas de frecuencia, según la naturaleza y distribución de las variables. ${ }^{20,21}$ 
El análisis bivariado permitió establecer la asociación de la higiene oral(evaluada en forma categórica) con cada una de las variables en el estudio, para cumplir con estos propósitos se aplicaron test de Chi cuadrado y test exacto de Fisher. ${ }^{20,21}$

Para el análisis multivariado se aplicó una análisis de regresión binomial, que estimó los RR (Riesgos Relativos: Razón de Prevalencia) y sus respectivos intervalos de confianza del $95 \% .{ }^{22,23}$ Se definió como variable dependiente higiene oral, categorizada como buena cuando el índice cuantificado de placa era inferior a 1 y mala cuando se obtenían valores mayores o iguales a 1 .

Como variables explicatorias se analizaron género, edad, institución educativa, grado escolar, higiene de la indumentaria, el cabello, manos y uñas, problemas de la piel y número de hábitos de autocuidado asumidos por el paciente, categorizado como bueno cuando asumía más de tres conductas de autocuidado, de las cinco evaluadas.

Para el análisis de regresión binomial bivariado inicial se analizó la variable dependiente con cada una de las variables explicatorias en forma independiente y a partir de este, se seleccionaron para el modelo final las variables que obtuvieron una probabilidad igual o inferior a $\mathrm{p}=0.25$ o aquellas que mostraban ser biológicamente significativas. ${ }^{24}$ Todo el análisis se realizó considerando un nivel de significancia de $\alpha=0.05$.

Todos los procedimientos siguieron las recomendaciones de la Resolución 008430 de 1993, ${ }^{25}$ del Ministerio de Salud, para el trabajo con menores de edad. Se solicitó la participación voluntaria tanto de la institución, de los padres, como del escolar en el estudio. Se preservó en todo momento la autonomía, la beneficencia, la confidencialidad y la privacidad de la información recolectada.

\section{RESULTADOS}

\section{Análisis Univariado}

Aspectos sociodemográficos

Se evaluaron 291 escolares; el 73.5\% eran del sexo femenino, el 53.6\% (156) estaban vinculados a la institución 2; el $60.8 \%$ (177) estaban en 1 y 2 grado de primaria y el $16.2 \%$ (47) vinculados a transición; el 77.3\% (225) de los escolares eran mayores de 6 años (Tabla 1). El promedio de edad en la población encuestada fue $7.02 \pm 1.74$ años, con rango de edad entre los 4 y 13 años.
Aspectos relacionados con el autocuidado

$\mathrm{Al}$ describir las condiciones relacionadas con hábitos de autocuidado se observó que el $84.2 \%$ (245) de la población tenía cuidado con su ropa, el 84.5\% (246) con su cabello, el 93.5\% (272) con su piel, el 74.9\% (218) con sus manos y uñas y el 7.9\% tenían buena higiene oral (Tabla 2). El promedio de hábitos de autocuidado adoptado por los escolares fue $3.44 \pm 0.97$ hábitos adoptados, con rangos entre 0 y 5 ; el promedio del índice cuantificado de placa fue de $1.93 \pm 0.64$, con rangos entre 0.16 y 3 .

\section{Análisis Bivariado}

Aspectos sociodemográficos y de higiene oral

$\mathrm{Al}$ analizar la población escolar de acuerdo a las variables sociodemográficas categorizado por buena y mala higiene oral se encontraron diferencias estadísticamente significativas para las variables grado escolar $(p=0.001)$ y edad $(p=0.003)$ Por grado escolar se observó mejor higiene oral en el grupo de transición en el $21.3 \%$ de los escolares, mientras que en el grupo de 3, 4 y 5 de primaria en el 1,5\% de los niños y las niñas (Tabla 1).

Por grupo de edad se observó que mientras el 16.7\% (11) de los escolares con edades iguales o inferiores a 6 años registraron buena higiene oral, sólo el 5.3\% (12) de los mayores de 6 años presentaron igual condición (Tabla1). El promedio de edad para los escolares con buena higiene oral fue de 5.95 años y en el de mala higiene oral 7.11 años.

Al comparar cada uno de los hábitos de autocuidado según higiene oral, sólo se determinó diferencia estadísticamente significativa $(\mathrm{p}=0.001)$ para la variable número de hábitos categorizada pues mientras el 11.9\% (21) escolares que adoptaban más de tres hábitos de autocuidado tenían buena higiene oral $1.7 \%$ de los que adoptaban 3 ó menos hábitos de autocuidado registraban una adecuada higiene dental (Tabla 2).

\section{Análisis Multivariado}

Análisis de la higiene oral según variables sociodemográficos y de autocuidado

$\mathrm{Al}$ intentar asociar la buena higiene oral con variables sociodemográficas, hábitos de autocuidado y número de hábitos de autocuidado adoptados por los escolares en forma no ajustada, se encontraron Riesgos Relativos (R.R.) asociados a tener buena higiene oral para ser hom- 
Tabla 1. Descripción del nivel de higiene oral considerando variables sociodemográficas.

\begin{tabular}{|c|c|c|c|c|}
\hline VARIABLE & $\begin{array}{c}\text { Global } \\
\text { Frecuencia (\%) }\end{array}$ & $\begin{array}{l}\text { Buena higiene } \\
\text { Frecuencia }[\%]\end{array}$ & $\begin{array}{l}\text { Mala higiene } \\
\text { Frecuencia }(\%)\end{array}$ & $\mathbf{P}^{*}$ \\
\hline $\begin{array}{l}\text { Población } \\
\text { Género }\end{array}$ & $291(100)$ & $23(7.9)$ & 268 (92.1) & \\
\hline Femenino & $214(73.5)$ & $16(7.5)$ & $198(92.5)$ & 0.629 \\
\hline $\begin{array}{l}\text { Masculino } \\
\text { Institución }\end{array}$ & $77(26.5)$ & $7(9.1)$ & $70(90.9)$ & \\
\hline Institución 1 & $135(46.4)$ & $14(10.4)$ & $121(89.6)$ & 0.147 \\
\hline $\begin{array}{l}\text { Institución } 2 \\
\text { Grado escolar }\end{array}$ & $156(53.6)$ & $9(5.8)$ & 147 (94.2) & \\
\hline Transición & $47(16.2)$ & $10(21.3)$ & $37(78.7)$ & $0.001^{4 *}+$ \\
\hline $\begin{array}{l}1^{0}-2^{\circ} \\
3^{\circ}-4^{0}-5^{0}\end{array}$ & $\begin{array}{c}177(60.8) \\
67(23)\end{array}$ & $\begin{array}{l}12(6.8) \\
1(1.5)\end{array}$ & $\begin{array}{l}165(93.2) \\
66(98.5)\end{array}$ & \\
\hline $\begin{array}{l}\text { Edad } \\
\leqslant 6 \text { años } \\
>6 \text { años }\end{array}$ & $\begin{array}{c}66(22.7) \\
225(77.3)\end{array}$ & $\begin{array}{l}11(16.7) \\
12(5.3)\end{array}$ & $\begin{array}{r}55(83.3) \\
213(94.7)\end{array}$ & $0.003+$ \\
\hline
\end{tabular}

Tabla 2. Descripción de los hábitos de autocuidado asumidos por los escolares descritos en forma Global y según higiene oral.

\begin{tabular}{|c|c|c|c|c|}
\hline VARIABLE & $\begin{array}{c}\text { Global } \\
\text { Frecuencia (\%) }\end{array}$ & $\begin{array}{l}\text { Buena higiene oral } \\
\text { Frecuencia }(\%)\end{array}$ & $\begin{array}{l}\text { Mala higiene oral } \\
\text { Frecuencia }(\%)\end{array}$ & $\mathbf{P}^{* *}$ \\
\hline $\begin{array}{l}\text { Población } \\
\text { Ropa }\end{array}$ & $291(100)$ & $23(7.9)$ & $268(92.1)$ & \\
\hline $\begin{array}{l}\mathrm{Si} \\
\mathrm{No}\end{array}$ & $\begin{array}{c}245(84.2) \\
46(15.8)\end{array}$ & $\begin{array}{l}21(8.6) \\
2(4.4)\end{array}$ & $\begin{array}{l}224(91.4) \\
44(95.6)\end{array}$ & 0.550 \\
\hline $\begin{array}{l}\text { Cabello } \\
\text { Si } \\
\text { No }\end{array}$ & $\begin{array}{c}246(84.5) \\
45(15.5)\end{array}$ & $\begin{array}{c}22(8.9) \\
1(2.2)\end{array}$ & $\begin{array}{c}224(91.1) \\
44(97.8)\end{array}$ & 0.224 \\
\hline $\begin{array}{l}\text { Piel } \\
\text { Si } \\
\text { No }\end{array}$ & $\begin{array}{c}272(93.5) \\
19(65)\end{array}$ & $\begin{array}{c}22(8.1) \\
1(5.3)\end{array}$ & $\begin{array}{c}250(91.9) \\
18(94.7)\end{array}$ & 1.0 \\
\hline $\begin{array}{l}\text { Manos - แñas } \\
\text { Si } \\
\text { No }\end{array}$ & $\begin{array}{c}218(74.9) \\
73(25.1)\end{array}$ & $\begin{array}{c}20(9.2) \\
3(4.1)\end{array}$ & $\begin{array}{c}198(90.8) \\
70(95.9)\end{array}$ & 0.214 \\
\hline $\begin{array}{l}\text { Hábitos de al } \\
>3 \text { hábitos } \\
\leqslant 3 \text { hóbitos }\end{array}$ & 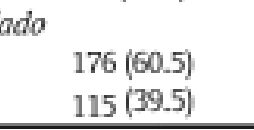 & $\begin{array}{c}21(11.9) \\
2(1.7) \\
\end{array}$ & $\begin{array}{l}155(88.1) \\
113(98.3)\end{array}$ & $0.001 *$ \\
\hline$\alpha=0.05$ & est exacto de Fis & * Estadistica & tte significativa & \\
\hline
\end{tabular}

bres (R.R. 1.21 IC95\% 0.52-2.8), Cuidado con la ropa (R.R. 1.97 IC95\% 0.47-8.12), Cuidado del cabello (R.R. 4.02 IC95\% 0.55-29.1, cuidado de manos y uñas (R.R. 2.23 IC95\% 0.687.3) y más de 3 hábitos de autocuidado adoptados por los escolares (R.R. 6.86 IC95\% 1.64-28.7); sin embargo, sólo esta última mostró diferencias estadísticamente significativas $(\mathrm{p}=0.008)$ (Tabla 3$)$.
Se encontraron como factores asociados a no tener buena higiene oral estar vinculados a la institución 2 (R.R. 0.55 IC95\% 0.25-1.24), estar matriculado en 1 y 2 grado de primaria (R.R. 0.31 IC95\% 0.15-) o en 3, 4 y 5 de primaria (R.R. 0.07 IC95\% 0.009-0.53), de estos alcanzaron diferencias estadísticamente significativas estar vinculado a 1 y 2 grado de primaria y a 3,4 y 5 de primaria $(p=0.004$ y 0.001 respectivamente) (Tabla 3). 
Tabla 3. Riesgos Relativos no ajustados por factores asociodos con buena higiene oral.

\begin{tabular}{|c|c|c|c|}
\hline FACTORES ASOCIADOS & R.R. & IC 95\% & $\mathbf{P}$ \\
\hline $\operatorname{Sen} 0$ & & & \\
\hline $\begin{array}{l}\text { Hombres } \\
\text { Institucion }\end{array}$ & 1.21 & $0.52 \cdot 2.8$ & 0.652 \\
\hline $\begin{array}{l}\text { Institución } 2 \\
\text { Grado escoier }\end{array}$ & 0.55 & $0.25-1.24$ & 0.153 \\
\hline $1^{2} \cdot 2^{2}$ & 0.31 & $0.15-0.69$ & $0.004^{*}$ \\
\hline $3^{2}-4^{e}-5^{n}$ & 0.07 & $0.009 \cdot 0.53$ & $0.001^{*}$ \\
\hline $\begin{array}{l}\text { Edad } \\
>6 \text { años }\end{array}$ & 0.32 & $0.15-0.69$ & $0.004^{*}$ \\
\hline Hábitos de autocuidado & & & \\
\hline Ropa & 1.97 & $0.47-8.12$ & 0.347 \\
\hline Cabello & 4.02 & $0.55-29.1$ & 0.168 \\
\hline Piel & 1.53 & $0.21-10.8$ & 0.666 \\
\hline Manos - uños & 223 & $0.68-7.3$ & 0.148 \\
\hline Más de tres bábitos & 6.86 & $1.64-28.7$ & $0.008^{*}$ \\
\hline
\end{tabular}

Tabla 4. Riesgos Relativos ajustados por factores asociados con buena higiene oral.

\begin{tabular}{lccc}
\hline FACTORES ASOCIADOS & R.R. & IC 95\% & P \\
\hline Más de tres hábitos & 14.4 & $3.47-59.9$ & $<0.0001^{*}$ \\
$1^{10}-2^{0}$ & 0.46 & $0.21-1.03$ & $0.059^{* *}$ \\
$3^{0}-4^{0}-5^{\circ}$ & 0.09 & $0.012-0.7$ & $0.022^{*}$ \\
Institución 2 & 0.45 & $0.2-1.01$ & $0.054^{* *}$ \\
\hline
\end{tabular}

"Estadisticamente significativos

"Marginalmente significativos

El modelo de regresión binomial ajustado por todas las variables mostró que adoptar más 3 hábitos de autocuidado con R.R. de 14.4 IC95\% 3.47-59.9 ( $\mathrm{P}<0.0001)$ estaba asociado a tener buena higiene oral. Sin embargo, el análisis también mostró que estar vinculado a 1 y 2 de primaria con R.R. 0.46 (IC95\% 0.21-1.03) y a los grados escolares de 3,4 y 5 de primaria con R.R. 0.09 (IC95\% 0.0120.7), y pertenecer a la institución 2 con R.R. 0.45 (IC95\% $0.2-1.01$ ) permanecieron como factores relacionados a no tener buena higiene oral con niveles de significancia de $\mathrm{p}=0.059, \mathrm{p}=0.022, \mathrm{p}=0.054$ respectivamente (Tabla 4).

\section{DISCUSIÓN}

Los resultados de la presente investigación, cuyos datos fueron recolectados por estudiantes de la Facultad de Odontología, en 291 escolares de dos instituciones educativas de Bucaramanga y su área metropolitana, con rango de edad entre los 4 y 13 años, mostraron que el adoptar más de tres hábitos de autocuidado general se relacionó con buena higiene oral.
Las intervenciones con una visión positiva e integral de la salud y dirigidas a las comunidades vulnerables tienen impacto no sólo en la población beneficiaria, sino que también le aporta a los estudiantes de odontología que se están formando y les ayuda a comprender las necesidades reales de la comunidad y el compromiso social que ellos y las universidades tienen frente a la necesidad de participar en la resolución de este tipo de situaciones. ${ }^{26} \mathrm{La}$ presente investigación, se desarrolló dentro de la asignatura de promoción de la salud y prevención de la enfermedad y permitió a los jóvenes llevar a la práctica conceptos de salud integral, promoción y de educación para la salud fundamentado en un diagnóstico de las necesidades de la comunidad sobre la cual iban a intervenir, creando espacios para el desarrollo de un programa con pertinencia social.

Un estudio comparativo, llevado a cabo por la OPS en 20 países de Latinoamérica y del Caribe, reveló que el contenido y la metodología educativa, de programas dirigidos a promover la salud en los escolares se basan exclusivamente en los daños a la salud, en lugar de enseñar hábitos sanos que se pudieran ampliar fuera del ámbito escolar y que esto podría explicar en parte, el escaso impacto de este tipo de programas. ${ }^{5}$ Esta afirmación y las evidencias que se presentan en este trabajo, corroboran la importancia de identificar los hábitos de autocuidado y enfatiza en la necesidad de integrar acciones en pro del escolar con el ánimo de generar un verdadero impacto en términos de salud y bienestar.

En la presente investigación, no se relacionó una buena higiene oral con un hábito de autocuidado en particular, como si lograron establecer Kassak y colaboradores, ${ }^{11}$ pues en su investigación mostraron que hábitos dietarios y el ejercicio físico adecuados se correlacionaban significativamente con la frecuencia de cepillado de los dientes en adolescentes, los mencionados investigadores resaltan, sin embargo, que una de las debilidades de su estudio radicaba en el autoreporte de estos comportamientos de salud y las posibles repercusiones de la deseabilidad social en las respuestas.

En este trabajo, se controló esta situación, a través de mecanismos de observación y registro por parte del encuestador; se evitó interrogar al escolar dada su edad (preescolar y escolar) y las posibles repercusiones de la 
deseabilidad social que podrían modificar los resultados, por esto no se consideró el autoreporte de hábitos dietarios y actividad fisica.

Encontrar un asociación 46\% mayor de mala higiene oral en el grupo de escolares matriculados en los niveles de 1 y 2 primaria, comparados con los de preescolar, tiene grandes repercusiones en el posible desarrollo de caries dental, pues el primer molar no ha entrado aun en oclusión y no hay mecanismo de autolimpieza asociado a la fricción que se produce durante la masticación, ${ }^{7,26}$ adicionalmente el diente recién erupcionado es más susceptible a la caries dental ya que el esmalte aun puede considerarse inmaduro, ${ }^{26}$ lo que hace necesario implementar acciones especiales orientadas a prevenir la caries dental en los niños y las niñas de primero y segundo de primaria de las instituciones investigadas.

Observar una mayor frecuencia de hábitos de autocuidado en preescolares se podría explicarse, por el hecho de que las mamás son, en la mayoría de los casos las responsables del cuidado corporal en los niños más pequeños, pues como lo expresan González y colaboradores, ${ }^{7}$ son las madres las principales transmisoras de los hábitos de salud y las creadoras de patrones de comportamiento en los niños. Estos resultados podrían indicar que es necesario que las madres acompañen a los escolares en su cuidado corporal hasta que estos cumplan los siete u ocho años o hasta que se logre verificar la autoeficacia en la adopción de estos hábitos.

Una buena higiene oral fue evidente en el 7.9\% de los escolares y fue el hábito de autocuidado adoptado con menor frecuencia por los menores, si se compara con buenos hábitos de higiene de la indumentaria, del cabello, manos y uñas y problemas de la piel que fueron asumidos por más del $75 \%$ de los escolares.

Cabe destacar el hecho de que para el único, de los hábitos analizados, que se empleó un índice que cuantificaba el nivel, fue para higiene oral; mientras que para las otras variables se definieron criterios que los cualificaban como adecuados 0 inadecuados, hecho que podría explicar las diferencias tan marcadas.

Otra consideración importante, es que no se controló por nivel educativo de la madre y otras variables que podrían confundir los resultados; sin embargo, la ventaja de fundamentarse en la observación de los hábitos, radi- can en que ésta fue igual, tanto para los escolares con buenos como con malos hábitos de autocuidado y no se esperaría diferencias significativas para uno y otro grupo en cuanto a las variables de confusión, así que se podría considerar que los factores de confusión se encontraba presentes en forma no diferencial en cada uno de los grupos lo que haría pensar que los riesgos relativos tenderían al valor nulo. Cabe considerar en próximas investigaciones ajustar por posibles factores de confusión y comparar los resultados.

Se podría concluir, entonces, que el abordaje de programas de promoción de la salud, que consideran la relación de el número hábitos de autocuidado general adoptados por los escolares con el hábito de higiene oral, puede constituirse en una estrategia que permite integrar acciones que fomentan la construcción de una cultura de la salud y que permite abordar el concepto de personas sanas más que bocas sanas, pues es este en últimas, el factor que nos motiva a implementar este tipo de acciones y que podrían mostrar mejores resultados en los programas orientados a promover la salud oral y que se realizan en las instituciones educativas. Adicionalmente, es importante mostrar en la práctica la importancia de este tipo de abordajes, en los procesos de enseñanza aprendizaje de los futuros profesionales de la salud oral.

\section{Agradecimientos}

Es fundamental agradecer el excelente trabajo realizado por los estudiantes de I semestre de promoción y prevención en la Facultad de Odontología de la Universidad Santo Tomás, que hicieron posible el desarrollo de la presente investigación.

\section{BIBLIOGRAFÍA}

1. Sheiham A, Watt RG. The common risk factor approach: A rational basis for promoting oral health. Community Dent Oral Epidemiol 2000; 28: 399 - 406.

2. Atwood K, Colditz GA, Kawachi I. From public health science to prevention police: placing science in its social political contexts. Am J Public Health 1997; 87: 1603 - 1606.

3. Moyses ST, Moyses SJ, Watt RG, Sheiham A. Associations between health promoting schools's policies and indicators of oral health in Brazil. Health Promot Int 2003; 18: 209 - 218.

4. Delgado MC. Influencia de un programa motivacional en niños campesinos entre siete y doce años de edad. CES Odontología 1996; 9: $29-34$. 
5. Revista Panamericana de Salud Pública. Temas de actualidad. Componentes educativos de los programas para la promoción de la salud escolar. Rev Panam Salud Pública 1997; 3: 209 - 214.

6. McGinnis JM. A vision for health in our new century. Am J Health Promot 2003; 18: 146 -150.

7. González MC, Valbuena LF, Zarta OL, Martignon S, Arenas M, Leaño M. Caries Dental. Guías de práctica clínica basadas en la evidencia. Bogotá: ACFO-ISS; 1998. p. 26 - 30.

8. Arias MI. Influencia de un video motivacional sobre las prácticas de higiene oral de niños entre los ocho y doce años. CES Odontología 1991; 4: 25 - 30.

9. Ylöstalo PV, Ek E, Laitinen J, Knuuttila ML. Optimism and life satisfaction as determinants for dental and general health behavior Oral health habits linked to cardiovascular risk factors. J Dent Res 2003; 82: 194 - 199.

10. Koivusilta L, Honkala S, Honkala E, Rimpelä A. Toothbrushing as part of the adolescent lifestyle predicts educational level. J Dent Res 2003; $82: 361$ - 366.

11. Kassak KM, Dagher R, Doughan B. Oral hygiene and lifestyle correlates among new undergraduaduate university students in Lebanon. J Am Coll Health 2001; 50: 15 - 20.

12. Kelsey, JL, Douglas TH. Methods in observational epidemiology. N.Y. Oxford University Press, 1986 Cap. 10 (Cross-Sectional another types of studies) p. 244 - 257.

13. Feinstein AR. Clinical biostatistics. XLII. The architecture of crosssectional research (Part I). Clin Pharmacol Ther 1978; 23: 81 - 96.

14. Feinstein AR. Clinical biostatistics. The architecture of crosssectional research (Conclusion). Clin Pharmacol Ther 1978; 481 - 493.

\section{CDC-OMS. Epi-Inf 6 Versión 6.04a Nov 1996}

16. República de Colombia. Estudio Nacional de Salud Bucal-ENSAB III. Colombia: Ministerio de Salud. Tomo VII, 1999.

17. Pieschacón MP, Camargo DM. Acuerdo entre el índice de Green y Vermillion con el índice cuantificado de placa. Ponencia XI Encuentro Nacional de Investigación Odontología. CES. Medellín, 2000.

18. Microsoft Excel 5.0, Microsoft Corporation 1997.

19. Stata corp. 2003. Stata statistical software. Release 8.0 College station, T.X: Stata Corporation.

20. Pagano M, Gauvreau K. Principles of Biostatistics. Duxbury Press. Belmont p. 35 - 44, 257 - 263.

21. Norman GR, Streiner DL. Bioestadística. Mosby/Doyma Libros: Madrid; 1996 p 129 - 147.

22. Wacholder S. Binomial regresión in GLIM: estimating risk and risk differences. Am J Epidemiol 1986; 123: 174 - 184.
23. Zochetti C, Consonni D, Vertáis PA. Relationship between prevalence rate ratios and odds ratios in cross-sectional studies. Int J Epidemiol 1997; 26: 220 - 223.

24. Bautista LE. Métodos avanzados de análisis. Bucaramanga 2002.

25. República de Colombia. Ministerio de Salud. Resolución No. 008430 del 4 de octubre de 1993.

26. Lalumandier JA, Williams KA, Curd FM. Educating and treating school children impacts much more than the child. Am J Health Educ 2005; 36: 48 - 50.

27. Axelsson P. Diagnosis and risk prediction of dental caries, Vol 2. Quintessence books: Chicago; 2000, p. 153

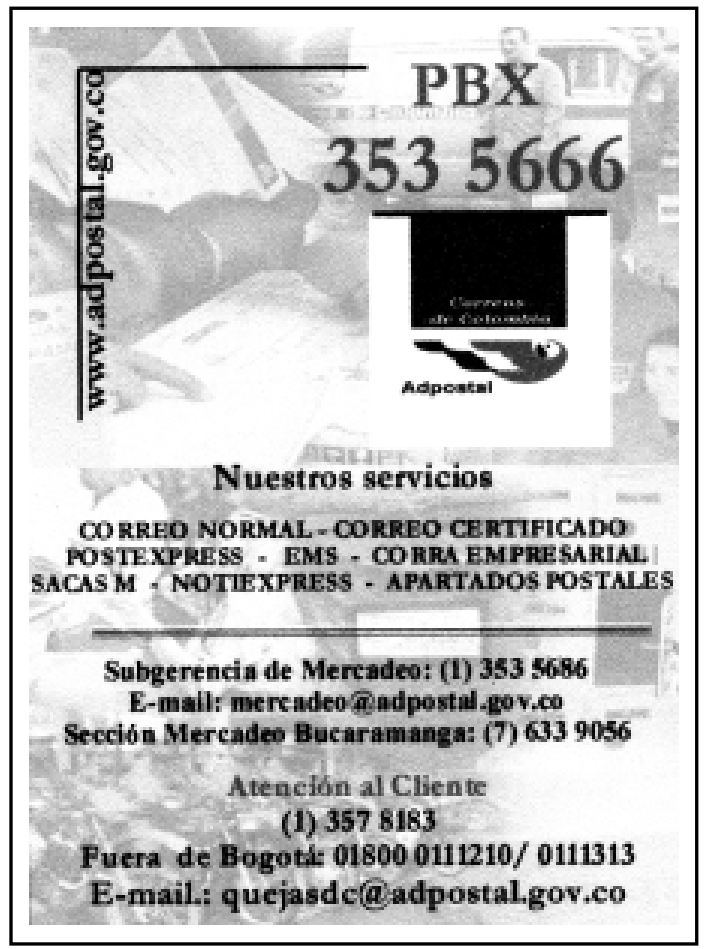

